\title{
Observation of spermidine-induced attractive forces in self-assembled monolayers of single stranded DNA using a microcantilever sensor
}

\author{
J. Mertens, ${ }^{\text {a) }}$ J. Tamayo, P. Kosaka, and M. Calleja \\ Institute of Microelectronics of Madrid (IMM-CNM), CSIC, Tres Cantos, 28760 Madrid, Spain
}

(Received 1 March 2011; accepted 31 March 2011; published online 13 April 2011)

\begin{abstract}
Despite the biological relevance, the physical origin of attraction between highly negatively charged DNA strands in condensation remains an open question. We have used microcantilever sensors to study the forces involved in DNA condensation by spermidine. The experiments were performed under flow conditions with gold-coated cantilevers sensitized with thiolated single stranded DNA. The experiments show that above a critical concentration of spermidine, the DNA strands abruptly experiences large attractive forces. The critical spermidine concentration for the transition increases with the monovalent salt concentration. The experiments provide a direct insight of the forces responsible of condensation. (C) 2011 American Institute of Physics. [doi:10.1063/1.3580775]
\end{abstract}

Genomic DNA is a very long molecule that behaves as wormlike coil in dilute solution due to its small persistence length. Its storage in the cell nucleus, bacterial nucleoids, and viral capsids in a compact ordered configuration requires a volume reduction between three and four orders of magnitude, depending on the organism. ${ }^{1,2}$ In the condensed state, the DNA segments are locally aligned and separated by one or two layers of water. The compaction plays an essential role in life, ${ }^{1}$ and can be exploited for the transport of DNA vectors in gene therapy. ${ }^{3}$ Naturally, DNA condensation is not a priori a process energetically favorable as it requires of a significant loss of configurational entropy, a high cost in bending mechanical energy, and it must overcome the high electrostatic repulsion between DNA strands (DNA is one of the most charged polymers with one negative charge per $0.17 \mathrm{~nm}$ of length). ${ }^{1,2}$ Therefore, the condensation process requires condensing agents, such as cationic proteins and polyamines. In particular, spermidine (Spd), $\left[\mathrm{H}_{3} \mathrm{~N}\left(\mathrm{CH}_{2}\right)_{3} \mathrm{NH}_{2}\left(\mathrm{CH}_{2}\right)_{4} \mathrm{NH}_{3}\right]^{3+}$, is a polyamine abundant in living cells that plays a key role in maintaining cellular DNA in a compact state. In addition it facilitates the packaging of double-stranded DNA in certain viruses. The molecular mechanism of the polyamine function in DNA condensation is presumed to involve the neutralization of the net charge of the DNA by the positively charged amino groups to a value about $10 \% .^{4-7}$ At this point, there is still a considerable energy barrier to achieve the condensation. Correlation forces between the polycations bound to the DNA, perturbation of the hydration near the DNA and the bound polycations, Debye-Hückel interactions, and van der Waals attractive forces have been proposed as possible mechanisms to condense DNA. However, the interaction forces that assist in this process remains poorly understood.

Surface stress based nanomechanical biosensors are a unique tool for measuring biomolecular interactions and molecular conformational changes with no need of molecular labeling. ${ }^{8-11}$ In these devices, molecular receptors that specifically interact with their ligand are immobilized on one side of nanomechanical system that usually is a microcantilever. The interactions between the receptors/ligand com-

${ }^{\text {a)} E l e c t r o n i c ~ m a i l: ~ j m e r t e n s @ i m m . c n m . c s i c . e s . ~}$ plexes with themselves, and with the cantilever surface generate in-plane forces that deflect the cantilever in the nanometer regime. The cantilever deflection can be sensitively detected by either optical or electrical techniques. This sensing concept has been applied for label-free detection of nucleic acids, proteins, and biomarkers. ${ }^{12-17}$ In addition, a change in the molecular environment that surrounds the molecules anchored to the cantilever can also induce a significant cantilever deflection that can be monitored in real time. This principle has been applied for measuring conformational changes in DNA molecular motors fueled by protons ${ }^{18}$ and for measuring the conformation change in highly packed DNA films induced by water intercalation. ${ }^{19}$ The molecular mechanism of single stranded DNA (ssDNA) immobilization on gold has been extensively studied with these devices and showed a complex mechanism of surface reorganization of the anchored molecule resulting from attractive and repulsive force between DNA strands. ${ }^{20}$

Here we have applied a surface stress nanomechanical sensor to study the interaction between a self-assembled monolayer of ss 16 mer DNA and the trivalent Spd cation.

Uncoated monocrystalline silicon microcantilever arrays were purchased from Mikromasch. Microcantilevers were $400 \mu \mathrm{m}$ long, $100 \mu \mathrm{m}$ wide, and $1.0 \mu \mathrm{m}$ thick [Fig. 1(a)]. The cantilevers were coated by sputtering evaporation with a $20 \mathrm{~nm}$ gold layer on top of a $2 \mathrm{~nm}$ adhesion layer of chromium. Freshly coated microcantilevers were incubated with $1 \mu \mathrm{M}$ of the thiolated ssDNA probe diluted in phosphate buffered saline (PBS) at $p \mathrm{H} 7.4$ for $48 \mathrm{~h}$ in order to immobilize a densely packed DNA layer with surface density on the order of $10^{13}$ molecules per $\mathrm{cm}^{2}$. Afterwards, the cantilevers were rinsed with PBS buffer and Milli-Q water to discard unspecific interactions. The oligonucleotide sequence

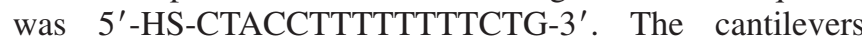
were placed in a flow-through glass cell with a volume of $100 \mu$ l. The liquid flow was controlled using a syringe pump (Cole Parmer) equipped with a low pressure injector valve and injection loop. We have used a flow rate of $3 \mathrm{ml} / \mathrm{h}$. The measurement of the cantilever deflections was carried out by a commercial readout system (MecWins) based on the optical beam deflection and the laser beam steering to measure several cantilevers sequentially ${ }^{21,22}$ [Fig. 1(b)]. Since the cantilever bending is affected by unwanted signals such as 


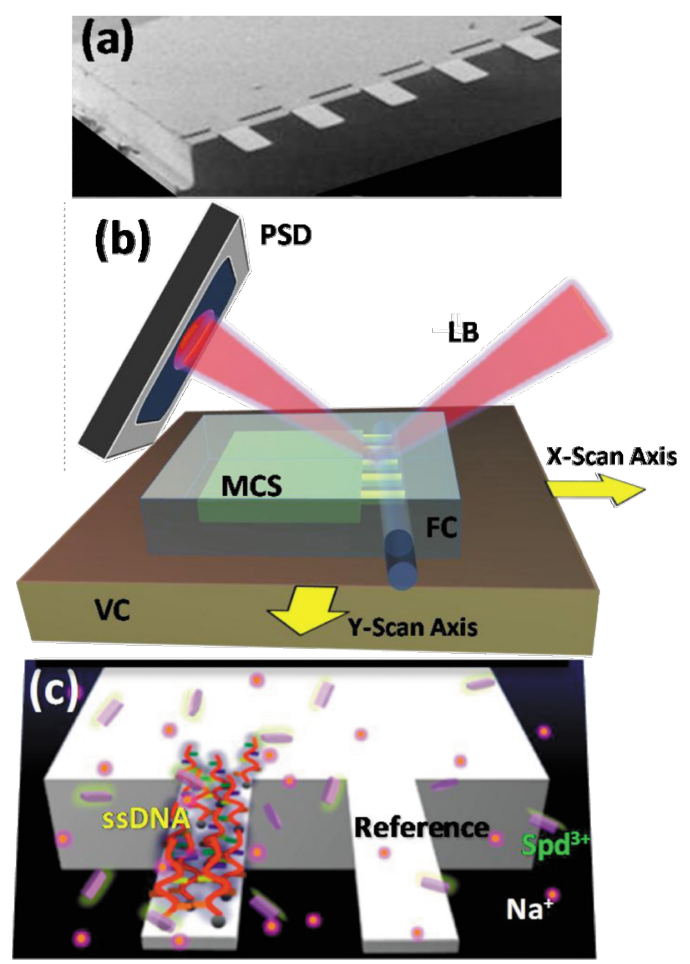

FIG. 1. (Color online) (a) Scanning electron microscopy image of the silicon cantilever arrays used in this letter. The nominal cantilever length, width, and thickness are $400 \mu \mathrm{m}, 100 \mu \mathrm{m}$, and $1 \mu \mathrm{m}$, respectively. (b) Schematic of the optical beam deflection system for readout of the cantilevers in solution. The following component labels are used: LB, laser beam; PSD, position sensitive detector; FC, fluid cell; MCS, microcantilevers; VC, voice-coil scanner. (c) Cartoon of the experimental method. The picture shows two microcantilevers in buffer solution with trivalent $\left(\mathrm{Spd}^{+3}\right)$ and monovalent cations $\left(\mathrm{Na}^{+}\right)$. The cantilever at the left is functionalized with SH-ssDNA, whereas the other cantilever is used as a reference.

nonspecific adsorption, small temperature variations, and refractive index changes, we acquired differential measurements using an in situ reference gold-coated cantilever to reject the nonspecific signals ${ }^{17}$ [Fig. 1(c)]. We use "bending up" to define the cantilever bending toward the gold side; whereas "bending down" refers to the bending toward the silicon side.

Figure 2(a) shows the absolute deflection signals measured for both the reference and the DNA-coated microcantilever after injection of $10 \mathrm{mM}$ of Spd in $1 \mathrm{M} \mathrm{NaCl}$ PBS buffer. Notice that the buffer ionic strength and $p \mathrm{H}$ were kept constant during the experiment. The Spd solution was injected at $\mathrm{t}=0$. In our setup, the time that requires the solution to pass through the tubing and fill the fluid cell is of about $200 \mathrm{~s}$. Then, the reference cantilever bent downwards (compressive surface stress on the gold and/or tensile surface stress on the silicon) until reaching a maximum bending at $\mathrm{t}=300 \mathrm{~s}$, then it bent up to recover the baseline before the injection at $t=600 \mathrm{~s}$. Since the silicon is negatively charged and the gold is positively charged at neutral $p \mathrm{H},{ }^{23}$ we relate the reference cantilever signal to the electrostatic binding of the Spd to the silicon surface. The DNA-coated cantilever showed a similar behavior to the reference cantilever during the first $50 \mathrm{~s}$ after the Spd reached the fluid cell. Then, an abrupt transition occurred, and the cantilever bent upward, implying a sudden change from compressive to tensile stress when the Spd reached a specific concentration. The generated tensile surface stress indicates that large attractive forces

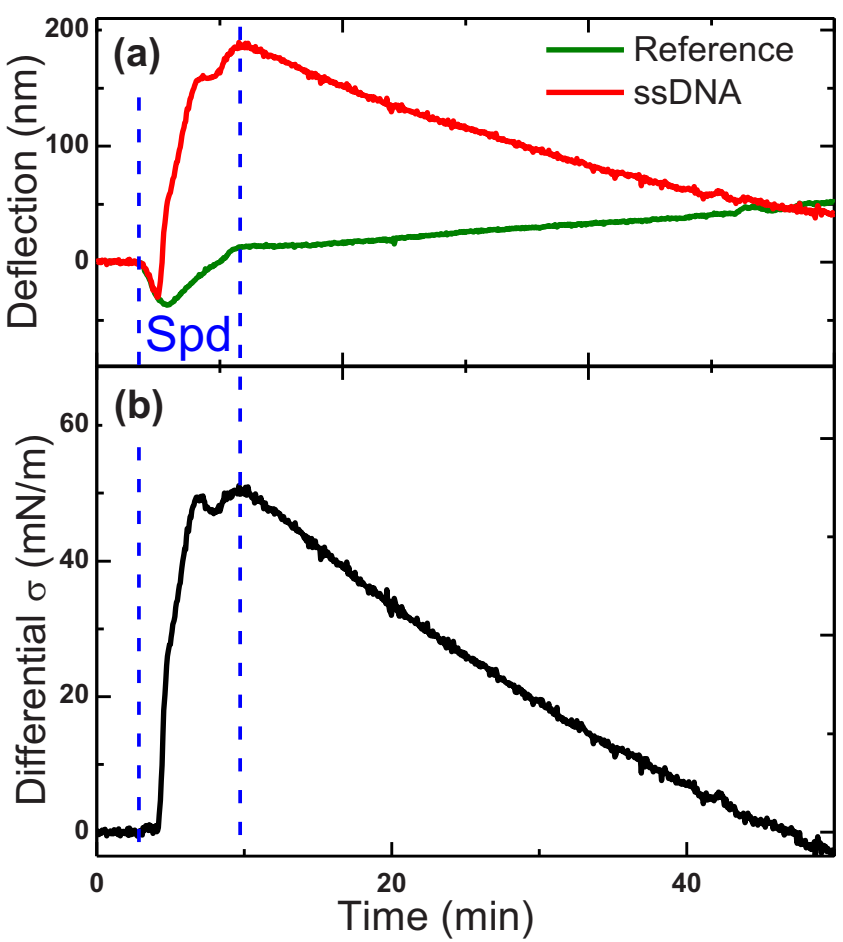

FIG. 2. (Color online) (a) Real-time deflection curves of the reference and DNA-sensitized cantilevers, when exposed to $10 \mathrm{mM}$ of Spd in PBS buffer with $1 \mathrm{M}$ of $\mathrm{NaCl}$. (b) Differential surface stress between the two cantilevers. The vertical dashed lines approximately indicate when the Spd solution reaches the fluid cell and when it is replaced by the buffer solution.

between the DNA strands anchored to the gold originate during this phase. This phenomenon can be more clearly observed in the differential surface stress signal plotted in Fig. 2(b). The nonmonotonic behavior of the bending signal and the presence of a double peak suggest a complex reorganization of the DNA - cations network during the attractive transition. The tensile stress induced by the binding of the Spd to the ssDNA is of about $50 \mathrm{mN} / \mathrm{m}$. By using a next-nearestneighbor interaction model for the surface stress, we estimate an attractive force between next nearest DNA molecules of 20-30 pN for grafting densities of $2-4 \times 10^{13} \mathrm{~cm}^{-2} \cdot{ }^{19}$ After the flush of the Spd solution by the PBS buffer, the cantilever recovered the bending level before the injection after $40 \mathrm{~min}$, approximately.

Previous experiments of three-dimensional DNA condensation in dilute solutions showed that the critical multivalent cation concentration, where the onset of aggregation occurs strongly depends on the concentration of the monovalent salt, usually $\mathrm{NaCl}^{1,5,24}$ The higher the salt concentration, the higher the multivalent cation concentration for the DNA condensation onset. Here, we have studied the surface stress induced by the Spd-DNA interaction for different concentrations of Spd and monovalent salt. Figure 3(a) shows the differential surface stress due to the DNA/Spd interaction for 10 $\mathrm{mM} \mathrm{Spd}$ at three PBS buffer ionic strengths that correspond to $0.1,1$, and $2 \mathrm{M}$ of $\mathrm{NaCl}$. For $0.1 \mathrm{M} \mathrm{NaCl}$, a large tensile stress (that is sign of significant attractive forces between DNA molecules) is quickly generated as the Spd flows over the cantilever. When the monovalent salt concentration is increased by ten times, attractive forces between the DNA molecules anchored to the cantilever are still observed. However, the magnitude of these forces has decreased between four and five times. If we further double the monovalent salt 

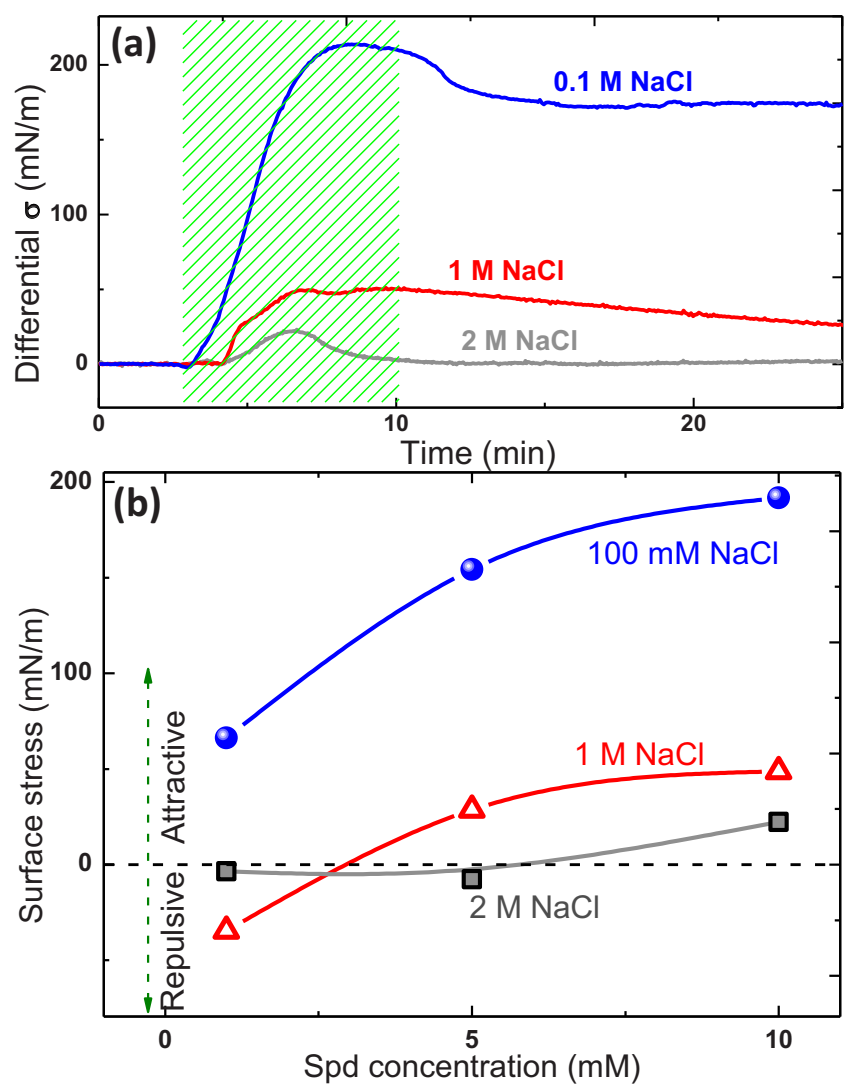

FIG. 3. (Color online) (a) Real-time differential surface stress induced by the $\mathrm{Spd} / \mathrm{ssDNA}$ interaction for $10 \mathrm{mM}$ of Spd and for $0.1,1$, and $2 \mathrm{M}$ of monovalent salt concentrations. The dashed region indicates the period during which the Spd is in the fluid cell. (b) Differential surface stress induced by the ssDNA/Spd interaction as a function of the Spd concentration for 0.1, 1 , and $2 \mathrm{M}$ of monovalent salt concentration. Positive surface stress indicates Spd-mediated attractive forces between ssDNA.

concentration, tensile stress is still generated but its amount is reduced 2.3 times. The results obtained here for twodimensional monolayers of short ssDNA follow the trend observed in three-dimensional aggregation, in which the competition between the multivalent and monovalent counterions increases the critical multivalent cation concentration for DNA precipitation. Moreover, a significant effect of the monovalent salt concentration is observed on the $\mathrm{Spd} / \mathrm{ssDNA}$ dissociation after the Spd solution has left the fluid cell. For the lowest salt concentration, the DNA film and the Spd form a stable complex that can last few hours. However, for the highest salt concentration, the Spd quickly dissociates and the cantilever recovers its original bending prior to the Spd injection.

Figure 3(b) summarizes the surface stress induced by the $\mathrm{Spd} / \mathrm{DNA}$ interaction as a function of the $\mathrm{Spd}$ and $\mathrm{NaCl}$ concentrations. The plotted values correspond to the surface stress at five minutes after the injection that is when the Spd concentration is maximum in the fluid cell. For $100 \mathrm{mM}$ $\mathrm{NaCl}$, the trivalent Spd cations are able to permeate between the monovalent counterions surrounding the DNA, and reverse the inter-DNA forces from repulsive to attractive. The magnitude of the attractive force increases with the Spd concentration. When the monovalent salt is increased to $1 \mathrm{M}$, the curve keeps the shape, but the attractive forces are largely reduced. Strikingly, repulsive forces are found for the lower Spd concentration, $1 \mathrm{mM}$. It seems that when the DNA molecules are surrounded by a dense monovalent counterion net- work, the introduction of Spd can initially induces repulsion between the DNA molecules due to the high osmotic pressure. For $2 \mathrm{M} \mathrm{NaCl}$, the monovalent counterions inhibit the condensation forces except for the highest Spd concentration $(10 \mathrm{mM})$, where a small tensile surface stress peak is detected.

In conclusion, we have shown that nanomechanical sensors can be added to the previously used biochemical, osmotic stress, x-ray scattering, and optical techniques to study the DNA condensation phenomena. Whereas the most of these techniques mostly study the three-dimensional DNA aggregation in solution, the nanomechanical sensor provides direct information about the forces involved in condensation in two-dimensional films. Notice that the molecules are restricted to their surface binding sites and the ssDNA strands are short, and then the configurational changes needed to achieve condensation are not allowed. Thus, the results give direct evidence that trivalent ions turn the repulsive electrostatic forces between short strands of single stranded DNA into attractive as a previous step to condensation.

This work was supported by grants from the Spanish Science Ministry through Projects Nos. TEC2009-14517C02, TRA2009-0117, and CSD2007-00010. The authors wish to acknowledge A. Cebollada for the gold deposition.

${ }^{1}$ V. A. Bloomfield, Curr. Opin. Struct. Biol. 6, 334 (1996).

${ }^{2}$ J. Kindt, S. Tzlil, A. Ben-Shaul, and W. M. Gelbart, Proc. Natl. Acad. Sci. U.S.A. 98, 13671 (2001).

${ }^{3} \mathrm{~S}$. Li and L. Huang, Gene Ther. 7, 31 (2000).

${ }^{4}$ K. Besteman, K. Van Eijk, and S. Lemay, Nat. Phys. 3, 641 (2007).

${ }^{5}$ V. A. Bloomfield, Biopolymers 31, 1471 (1991).

${ }^{6}$ X. Qiu, K. Andresen, J. S. Lamb, L. W. Kwok, and L. Pollack, Phys. Rev. Lett. 101, 228101 (2008).

${ }^{7}$ B. A. Todd, V. Adrian Parsegian, A. Shirahata, T. Thomas, and D. C. Rau, Biophys. J. 94, 4775 (2008).

${ }^{8}$ M. K. Ghatkesar, H. P. Lang, C. Gerber, M. Hegner, and T. Braun, PLoS ONE 3, e3610 (2008).

${ }^{9}$ M. L. Sushko, J. H. Harding, A. L. Shluger, R. A. McKendry, and M. Watari, Adv. Mater. (Weinheim, Ger.) 20, 3848 (2008).

${ }^{10}$ P. S. Waggoner and H. G. Craighead, Lab Chip 7, 1238 (2007).

${ }^{11}$ A. Boisen and T. Thundat, Mater. Today 12, 32 (2009).

${ }^{12}$ M. Alvarez, A. Calle, J. Tamayo, L. M. Lechuga, A. Abad, and A. Montoya, Biosens. Bioelectron. 18, 649 (2003).

${ }^{13}$ Y. Arntz, J. Seelig, H. Lang, J. Zhang, P. Hunziker, J. Ramseyer, E. Meyer, M. Hegner, and C. Gerber, Nanotechnology 14, 86 (2003).

${ }^{14}$ T. Braun, M. K. Ghatkesar, N. Backmann, W. Grange, P. Boulanger, L. Letellier, H. P. Lang, A. Bietsch, C. Gerber, and M. Hegner, Nat. Nanotechnol. 4, 179 (2009).

${ }^{15}$ J. W. Ndieyira, M. Watari, A. D. Barrera, D. Zhou, M. Vögtli, M. Batchelor, M. A. Cooper, T. Strunz, M. A. Horton, and C. Abell, Nat. Nanotechnol. 3, 691 (2008).

${ }^{16}$ G. Wu, R. H. Datar, K. M. Hansen, T. Thundat, R. J. Cote, and A. Majumdar, Nat. Biotechnol. 19, 856 (2001).

${ }^{17}$ J. Zhang, H. Lang, F. Huber, A. Bietsch, W. Grange, U. Certa, R. McKendry, H. J. Güntherodt, M. Hegner, and C. Gerber, Nat. Nanotechnol. 1, 214 (2006).

${ }^{18}$ W. Shu, D. Liu, M. Watari, C. K. Riener, T. Strunz, M. E. Welland, S Balasubramanian, and R. A. McKendry, J. Am. Chem. Soc. 127, 17054 (2005).

${ }^{19}$ J. Mertens, C. Rogero, M. Calleja, D. Ramos, J. A. Martín-Gago, C. Briones, and J. Tamayo, Nat. Nanotechnol. 3, 301 (2008).

${ }^{20}$ S. Kim, D. Yi, A. Passian, and T. Thundat, Appl. Phys. Lett. 96, 153703 (2010).

${ }^{21}$ J. Mertens, M. Álvarez, and J. Tamayo, Appl. Phys. Lett. 87, 234102 (2005).

${ }^{22}$ N. Martínez, P. Kosaka, J. Tamayo, J. Ramírez, O. Ahumada, J. Mertens, T. Hien, C. Rijn, and M. Calleja, Rev. Sci. Instrum. 81, 125109 (2010).

${ }^{23}$ P. M. Wolny, J. P. Spatz, and R. P. Richter, Langmuir 26, 1029 (2010).

${ }^{24}$ Y. Burak, G. Ariel, and D. Andelman, Biophys. J. 85, 2100 (2003). 